

\title{
Synthesis of 2-aryl-1,3-benzoselenazoles from bis(2- aminophenyl) diselenides and carboxylic acids using $\mathrm{PBu}_{3}$
}

\author{
Cátia S. Radatz (PG), ${ }^{1}$ Daniel S. Rampon (PG), ${ }^{1}$ Renata A. Balaguez (IC), ${ }^{2}$ Diego \\ Alves (PQ), ${ }^{2}$ Paulo H. Schneider (PQ ${ }^{* 1}$
}

1. Instituto de Química, Departamento de Química Orgânica, Universidade Federal do Rio Grande do Sul, UFRGS, 91501-970, Porto Alegre-RS, Brazil; 2. Instituto de Química e Geociências, LASOL, Universidade

Federal de Pelotas, UFPel, P.O. Box 354, 96010-900, Pelotas-RS, Brazil

*e-mail corresponding author: paulos@iq.ufrgs.br

Keywords: Benzoselenazoles, heterocycles, selenium, carboxylic acids.

\section{INTRODUCTION}

The investigation about different seleniumcontaining heterocyclic compounds has expanded rapidly during last years. ${ }^{1}$ Furthermore, substituted benzochalcogenazole systems have presented also interesting pharmaceutical properties. ${ }^{2}$ Besides, the synthesis of 2-substituted-1,3-benzothiazoles have attracted much attention according to their notably photophysical properties. ${ }^{3}$ Our continuing interest on the synthesis of organoselenium compounds ${ }^{4}$ prompted us to explore a general procedure to obtain 2-substituted 1,3-benzoselenazoles from bis(2-aminophenyl) diselenide (1a) and carboxylic acids (2a-m), using tributylphosphine in toluene under conventional heating or microwave irradiation (scheme 1).

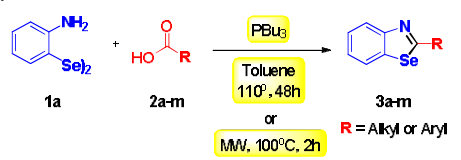

Scheme 1. Synthesis of 2-aryl-1,3-benzoselenazoles (3am) promoted by $\mathrm{PBu}_{3}$.

\section{RESULTS AND DISCUSSION}

In a first experiment, bis(2-aminophenyl) diselenide 1a (1.0 equiv), acid 4-bromobenzoic 2a (1.0 equiv) and tributylphosphine (1.0 equiv), were reacted in dichloromethane $(5 \mathrm{~mL})$ at room temperature. After 120 hours, the benzoselenazole 3a was obtained in only $10 \%$ yield. In order to improve the yield, several parameters were screened. At first a variety of solvents were evaluated under reflux temperature (MeCN, THF, toluene, xylene and DMSO $-120^{\circ} \mathrm{C}$ ). The best result afforded the product $3 \mathrm{a}$ in excellent yield (97\%) using toluene, 2.0 equiv of $1 \mathrm{a}$ and 3.0 equiv of $\mathrm{PBu}_{3}$ after 48 hours. Over recent years, it has been shown that the organic reactions can also be induce by microwave irradiation (MW), ${ }^{5}$ along with reduced reaction times and good yields. In this sense, the suitable reaction conditions were employed under microwave heating, affording the product $3 a$ in excellent yield (92\%) after only 2 hours (scheme 2). To explore the scope and limitations of this method, the reactions between a variety of carboxylic acids (2a-m) and 1a were investigated under the improved conditions (scheme 2).

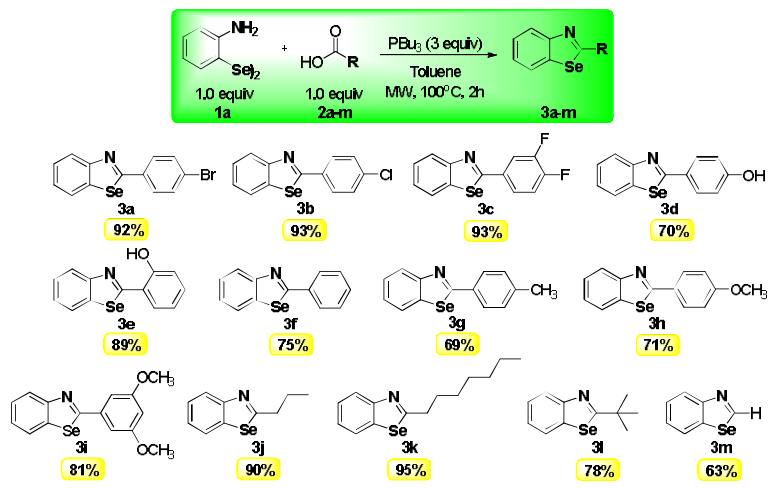

Scheme 2. Scope of the 2-substituted-1,3benzoselenazoles synthesis.

Currently, we are working on the study of a probable mechanism employing NMR ${ }^{77}$ Se and ${ }^{31} \mathrm{P}$, and also evaluating the effect of microwave heating in acceleration of reaction times.

\section{CONCLUSION}

In conclusion, we present here a general and easy method for the synthesis of several 2 substituted1,3-benzoselenazoles. The reaction afforded good to excellent yields after short reaction times under microwave irradiation. The mechanistic studies about this reaction are under evaluation.

\section{ACKNOWLEDGEMENTS}

CAPES, CNPq, INCT-Catalysis, FAPERGS, UFRGS and UFPel.

\section{REFERENCES}

${ }^{1}$ (a) Goodman, C. Nature Chem. Bio. 2013, 9, 214. (b) Koehnke, J.; et al. Chem. Bio. Chem. 2013, 5, 564 .

2 Tareq, M. et. al (Eds). Topics in Heterocyclic Chemistry, Bioactive Heterocycles III. Springer, Berlin, 2007.

${ }^{3}$ Gupta, A.; Rawat, S. J. Curr. Pharm. Res. 2010, 3, 13.

${ }^{4}$ Radatz, C.; et al. Tetrahedron. 2013, 691316.

${ }^{5}$ Kappe, C. O.; et. al (Eds.). Practical Microwave Synthesis for Organic Chemists: Strategies, Instruments, and Protocols. Wiley-VCH, Weinheim, 2009. 